\title{
Efficacy and safety of cisplatin and weekly docetaxel in patients with recurrent or metastatic squamous cell carcinoma of the head and neck
}

\author{
Moon Jin $\mathrm{Kim}^{1}$, Sung Min Kim², Hyun Ae Jung 3 , Jung Yong Hong ${ }^{4}$, Won Jin Chang ${ }^{5}$, Moon Ki Choi ${ }^{6}$, \\ Hye Sook Kim ${ }^{2}$, Jong-Mu Sun ${ }^{7}$, Keunchil Park ${ }^{7}$, and Myung-Ju Ahn ${ }^{7}$
}

\begin{abstract}
${ }^{1}$ Division of HematologyOncology, Department of Internal Medicine, Myongji Hospital, Goyang; ${ }^{2}$ Division of HematologyOncology, Department of Medicine, Samsung Changwon Hospital, Sungkyunkwan University School of Medicine, Changwon; ${ }^{3}$ Division of Hematology-Oncology, Hallym University Dongtan Sacred Heart Hospital, Hwaseong; ${ }^{4}$ Department of Oncology, Asan Medical Center, University of Ulsan College of Medicine, Seoul; ${ }^{5}$ Division of Hematology-Oncology, Department of Internal Medicine, Korea University College of Medicine, Seoul; ${ }^{6}$ Center for Colorectal Cancer, National Cancer Center, Goyang; ${ }^{7}$ Division of Hematology-Oncology, Department of Medicine, Samsung Medical Center, Sungkyunkwan University School of Medicine, Seoul, Korea
\end{abstract}

Received: July 1, 2017

Revised : December 8, 2017

Accepted: February 7, 2018

\author{
Correspondence to \\ Myung-Ju Ahn, M.D. \\ Division of Hematology- \\ Oncology, Department of \\ Medicine, Samsung Medical \\ Center, Sungkyunkwan \\ University School of Medicine, \\ 81 Irwon-ro, Gangnam-gu, Seoul \\ 06351, Korea \\ Tel: $+82-2-3410-3438$ \\ Fax: $+82-2-3410-1754$ \\ E-mail: silk.ahn@samsung.com
}

Background/Aims: We investigated the efficacy and toxicity of a weekly schedule of docetaxel and cisplatin as a first-line treatment in patients with recurrent or metastatic head and neck squamous cell carcinoma (R/M HNSCC).

Methods: In this study, 18 patients with previously diagnosed R/M HNSCC were treated with combination chemotherapy of weekly docetaxel $35 \mathrm{mg} / \mathrm{m}^{2}$ (day 1 and 8) and cisplatin $70 \mathrm{mg} / \mathrm{m}^{2}$ (day 1 ) as first-line chemotherapy, repeated every 3 weeks.

Results: Partial response and stable disease were observed in six patients $33.3 \%$; $95 \%$ confidence interval [CI], $11.1 \%$ to $55.6 \%$ ) and six patients (33.3\%; $95 \%$ CI, $11.1 \%$ to $55.6 \%)$, respectively. The median overall survival and progression-free survival were 11.26 months (95\% CI, 8.87 to 15.83) and 5.68 months ( $95 \%$ CI, 4.80 to 6.51), respectively. The major toxicity was grade $1 / 2$ anemia (50\%). Grade $3 / 4$ neutropenia was observed in one patient (5.6\%). Among the non-hematologic toxicities, grade $1 / 2$ hepatotoxicity was most common (22.2\%), and grade $3 / 4$ infection was observed in one patient (5.6\%). There was no treatment-related mortality.

Conclusions: For patients with R/M HNSCC, a cisplatin and weekly docetaxel regimen showed high efficacy with tolerable toxicity as a first-line treatment.

Keywords: Cisplatin; Docetaxel; Head and neck neoplasms; Carcinoma, squamous cell

\section{INTRODUCTION}

Head and neck squamous cell carcinoma (HNSCC) is the sixth most common cancer worldwide, with approximately 500,000 new cases diagnosed annually [1]. Most patients with HNSCC present with locally or regionally advanced disease that requires concurrent chemotherapy and radiotherapy or surgery $[2,3]$. However, approximately $10 \%$ of patients with HNSCC have distant metastasis at the time of initial presentation, and up to $20 \%$ of patients experience relapse in a site that requires chemotherapy. For these patients with recurrent or metastatic $(\mathrm{R} /$ M) HNSCC, platinum-based palliative chemotherapy is commonly used for first-line treatment, resulting in a median survival of 6 to 9 months and an 1-year survival rate of $40 \%$ [4].

Docetaxel, a semisynthetic taxoid derived from the rare Pacific yew tree 
Taxus baccata [5], is active against R/M HNSCC. The combination of docetaxel and platinum-based cytotoxic agents has led to a high response rate, ranging from $33 \%$ to $53 \%$, in previous studies [6-9]. However, owing to severe myelosuppression related to the 3-week dosing schedule of docetaxel, an alternative schedule of weekly docetaxel as a single agent has been evaluated in several studies, and showed comparable efficacy and acceptable toxicity in several cancers [10-14]. We previously reported that this combination regimen has an acceptable outcome as first-line chemotherapy in patients with recurrent or metastatic nasopharyngeal cancer, with an objective response rate (ORR) of $70.2 \%$ and a median overall survival (OS) of 28.5 months [14]. Kim et al. [15] also showed that a regimen of weekly docetaxel and cisplatin was active and tolerable as front-line treatment in advanced gastric cancer. In contrast, studies on the efficacy and toxicity of a platinum doublet with a weekly schedule of docetaxel have not yet been reported in $\mathrm{R} / \mathrm{N}$ HNSCC.

Therefore, we designed this single-center, retrospective study to evaluate the efficacy and toxicity of cisplatin and weekly docetaxel as first-line chemotherapy in the treatment of R/M HNSCC.

\section{METHODS}

\section{Patient eligibility}

We retrospectively collected and reviewed the medical records of patients with cytologically or histologically confirmed HNSCC. Other eligibility criteria included at least one measurable lesion according to Response Evaluation Criteria in Solid Tumors (RECIST) v1.o; age $>18$ years; Eastern Cooperative Oncology Group (ECOG) performance status o-2; and adequate hematologic function with absolute neutrophil count (ANC) > $1,500 / \mathrm{mm}^{3}$, platelet count $>100,000 / \mathrm{mm}^{3}$; adequate liver function with total bilirubin $<1.5 \mathrm{mg} / \mathrm{dL}$, aspartate aminotransferase, alanine aminotransferase $<2.5 \times$ upper limit of normal (ULN), alkaline phosphatase $<5 \times$ ULN in patients without liver metastasis; and adequate renal function with serum creatinine $<1.5 \mathrm{mg} / \mathrm{dL}$ or calculated creatinine clearance $>60$ by the Cockcroft-Gault method. Prior chemotherapy for recurrent or metastatic disease was not permitted. Patients with prior induction or adjuvant chemotherapy were eligible if at least 6 months had elapsed from their last course of chemotherapy. Other exclusion criteria included active infection, history of myocardial infarction in the last 3 months, uncontrolled congestive heart failure or hypertension, uncontrolled diabetes mellitus, history of prior chemotherapy with docetaxel, pregnancy, lactation, and other malignancy within 5 years. This study was approved by the Institutional Review Board of Samsung Medical Center (2014-02-049). All patients were drawn from existing research databases and had provided informed consent to be included in research studies on these treatments.

\section{Treatment}

Cisplatin was administered at a dose of $70 \mathrm{mg} / \mathrm{m}^{2}$ by intravenous infusion in $150 \mathrm{~mL}$ normal saline over 60 minutes on day 1 , and docetaxel was administered a dose of $35 \mathrm{mg} / \mathrm{m}^{2}$ by intravenous infusion in $100 \mathrm{~mL}$ dextrose water over 60 minutes on days 1 and 8 . All patients received adequate hydration, with at least 1,500 $\mathrm{mL}$ of half-normal saline or normal saline prior to cisplatin administration. Patients also received antiemetic therapy consisting of an intravenous $5-\mathrm{HT}_{3}$ antagonist and dexamethasone before docetaxel administration. The treatment cycles were repeated every 3 weeks for a maximum of six cycles.

Toxicities were evaluated according to the Common Terminology Criteria for Adverse Events (CTCAE), version 3.o. Patients whose absolute neutrophil and platelet counts were $\geq 1,500$ and $100,000 / \mathrm{mm}^{3}$, respectively, and who had grade 1 or less non-hematologic toxicity (excluding alopecia), received chemotherapy on day 1 of each cycle.

On days 1 and 8 of each cycle, the minimum requirements to receive chemotherapy were an ANC between 1,000 and $1,500 / \mathrm{mm}^{3}$, a platelet count $\geq 75,000 / \mathrm{mm}^{3}$ and no grade $\geq$ a non-hematologic toxicity (excluding alopecia). If there were any grade 3 or 4 hematologic toxicities at the nadir of the previous cycle, or febrile neutropenia with or without documented infection, then administration of both drugs in the subsequent cycles was reduced $20 \%$ from the planned dose. The administration of granulocyte-colony stimulating factor was allowed in the presence of febrile neutropenia or grade 3 or 4 neutropenia. In the presence of grade 3 or 4 
non-hematologic toxicity (except nausea, vomiting, and alopecia), the treatment was postponed until resolution of the toxicity and then both drug doses were reduced by $25 \%$ for the next cycle. In the case of grade 2 neurotoxicity, treatment was postponed until resolution of the toxicity to the level of grade 1 or less within 3 weeks; at that point, the doses of both drugs in subsequent cycles were reduced by $25 \%$ from the planned dose. In the presence of grade 2 asthenia, the subsequent cycle was also postponed until resolution to the level of grade 1 or less within 3 weeks; subsequent cycles were then performed with a $25 \%$ dose reduction from the planned dose of docetaxel. Treatment was stopped at any time for documented disease progression or unacceptable toxicity, or at the patient's request.

\section{Assessment of efficacy}

The pretreatment baseline evaluation included a complete medical history and physical examination, complete blood cell count (CBC) with differential, chemistry profiles, and performance status. Chest radiography, chest and upper abdominal computed tomography (CT) imaging, brain CT or magnetic resonance imaging (MRI), radionuclide bone scans, and other diagnostic procedures were performed as clinically indicated.

During treatment, a limited history, physical examination, assessment of toxicity, CBC with differential, and blood chemistry tests were repeated weekly. A chest radiograph was obtained every 3 weeks, prior to each cycle. Appropriate imaging studies, including CT scans of the chest and upper abdomen, were performed every two cycles to assess the treatment response, and sooner if required to document disease progression. Objective tumor responses were assessed according to the RECIST criteria using CT scan or MRI [16].

\section{Statistical analysis}

SPSS version 18.0 (SPSS Inc., Chicago, IL, USA) was used for statistical analysis. The response rate was calculated as the ratio of the number of patients who achieved a complete response (CR) or partial response (PR) to the number of enrolled patients. Progression-free survival (PFS) was defined as the time from the date of first administration of chemotherapy to the date of documented progression or loss to follow-up or death due to any cause. OS was defined as the time from the date of first administration of chemotherapy to the date of death or final follow-up. Response rate and patient clinical characteristics were evaluated using the chi-square test or Fisher's exact test. PFS and OS were calculated with the Kaplan-Meier method and the 95\% confidence interval (CI) for the median time to an event.

\section{RESULTS}

\section{Patient characteristics}

Between August 2007 and October 2013, a total of 18 patients were enrolled from a single center, Samsung Medical Center. Baseline patient characteristics are shown in Table 1. All 18 patients enrolled in the study, comprising 17 males (94.4\%) and one female (5.6\%), were included in an intent-to-treat analysis for the safety profile. One patient's response could not be evaluated owing to loss to follow-up after one cycle of chemotherapy. The median age of the patients was 63 years (range, 41 to 76 ). Seven patients (38.9\%) were older than 65 years. All 18 patients were former or current smokers. Subsequent chemotherapy was given to five patients who experienced progression following the study treatment. Only two patients received more than second-line chemotherapy. The most common site of metastasis was the lymph nodes $(\mathrm{n}=12)$, followed by lung $(\mathrm{n}=8)$, bone $(\mathrm{n}=5)$, and brain $(\mathrm{n}=1)$.

\section{Treatment administration}

A total of 61 cycles of chemotherapy were administered, with a median number of three cycles per patient (range, 1 to 6 ). Two patients (11.1\%) completed six cycles of chemotherapy as planned, two (11.1\%) received five cycles, four $(22.2 \%)$ received four cycles, and 10 (55.6\%) received fewer than four cycles. Dose reduction was required in two patients, and administration of docetaxel on day 8 was omitted in two patients. The main cause of dose reduction was grade 3 to 4 neutropenia.

\section{Treatment response and survival rate}

Among a total of 18 patients, 17 were evaluated for treatment response. In one patient, early discontinuation of the study occurred because of loss to follow-up before the first evaluation. As shown in Table 2, the ORR (CR + $\mathrm{PR})$ was $33.3 \%(6 / 18)$ and all six patients achieved PR 
Table 1. Patient characteristics

\begin{tabular}{|c|c|}
\hline Characteristic & Value \\
\hline Age, yr & $63(41-76)$ \\
\hline \multicolumn{2}{|l|}{ Sex } \\
\hline Male & $17(94.4)$ \\
\hline Female & $1(5 \cdot 6)$ \\
\hline \multicolumn{2}{|l|}{ ECOG PS } \\
\hline o & $1(5.6)$ \\
\hline 1 & $12(66.7)$ \\
\hline 2 & $5(27 \cdot 7)$ \\
\hline \multicolumn{2}{|l|}{ Primary site } \\
\hline Oral cavity & $5(27.7)$ \\
\hline Oropharynx & $2(11.1)$ \\
\hline Hypopharynx & $4(22.2)$ \\
\hline Larynx & $7(38.9)$ \\
\hline \multicolumn{2}{|l|}{ Initial disease status } \\
\hline Recurrent with distant metastasis & $3(16.7)$ \\
\hline Locally recurrent & $6(33 \cdot 3)$ \\
\hline Metastatic & $9(50.0)$ \\
\hline \multicolumn{2}{|l|}{ Prior therapy } \\
\hline Operation & $9(50.0)$ \\
\hline Adjuvant chemoradiotherapy & $9(50.0)$ \\
\hline Radiotherapy alone & $8(44 \cdot 4)$ \\
\hline Adjuvant chemotherapy & $1(5.6)$ \\
\hline \multicolumn{2}{|l|}{ No. of involved organs } \\
\hline 1 & $7(38.9)$ \\
\hline 2 & $5(27.8)$ \\
\hline 3 & $2(11.1)$ \\
\hline$\geq 4$ & $4(22.2)$ \\
\hline \multicolumn{2}{|l|}{ Metastatic sites } \\
\hline Lymph node & $12(66.7)$ \\
\hline Lung & $8(44.4)$ \\
\hline Bone & $5(27.8)$ \\
\hline Brain & $1(5.6)$ \\
\hline
\end{tabular}

Values are presented as median (range) or number (\%). ECOG PS, Eastern Cooperative Oncology Group performance status.

$33.3 \%$ (95\% CI, $11.1 \%$ to $55.6 \%$ ). Six patients (33.3\%) were confirmed to have stable disease. With a median follow-up duration of 26.8 months (range, 8 to 70.5 ), the median OS and PFS were 11.26 months (95\% CI, 8.87 to 15.83) (Fig. 1) and 5.68 months (95\% CI, 4.80 to 6.51) (Fig. 2), respectively.
Table 2. Treatment response

\begin{tabular}{lcc}
\hline Variable & No. of patients (\%) & \multicolumn{1}{c}{$95 \%$ CI } \\
\hline Best response & & \\
Complete response & 0 & 0 \\
\hline Partial response & $6(33.3)$ & $11.1-55.6$ \\
\hline Stable disease & $6(33.3)$ & $11.1-55.6$ \\
\hline Progressive disease & $5(27.8)$ & $5.6-50.0$ \\
\hline Not evaluable & $1(5.6)$ & $0-16.7$ \\
\hline Overall response rate & $6(33.3)$ & \\
\hline Disease control rate & $12(66.6)$ & \\
\hline
\end{tabular}

CI, confidence interval.

\section{Toxicity}

All patients were evaluated for toxicity. Table 3 shows the major hematologic and non-hematologic toxicities. Grade $1 / 2$ anemia was the most common toxicity (50\%). Grade 3/4 febrile neutropenia occurred in one patient (5.6\%), who experienced grade 4 neutropenia. Grade 3/4 neutropenia was observed in one patient (5.6\%). Among the non-hematologic toxicities, grade $1 / 2$ hepatotoxicity was the most common (22.2\%), and grade $3 / 4$ infection was observed in one patient (5.6\%). There was no treatment-related mortality.

\section{DISCUSSION}

In the present study, the combination of cisplatin and weekly docetaxel chemotherapy as first-line treatment of R/M HNSCC showed high efficacy, with a 33.3\% response rate and tolerable toxicity. These results are consistent with the findings of previous studies demonstrating a response rate of $33 \%$ to $53 \%$. It is of note that grade $1 / 2$ anemia was the major toxicity, observed in $50 \%$ of patients, but grade $3 / 4$ neutropenia was only found in one patient. Moreover, the median PFS was 5.6 months, and the median OS was 11.26 months, which is a quite encouraging finding.

Previously, a number of chemotherapeutic agents including platinum, taxanes, 5 -fluorouracil (5-FU), methotrexate, and ifosfamide have been demonstrated to have single-agent activity as first-line treatment for patients with R/M HNSCC [17,18]. In 1992, two studies established the use of platinum-based doublets with 5-FU 


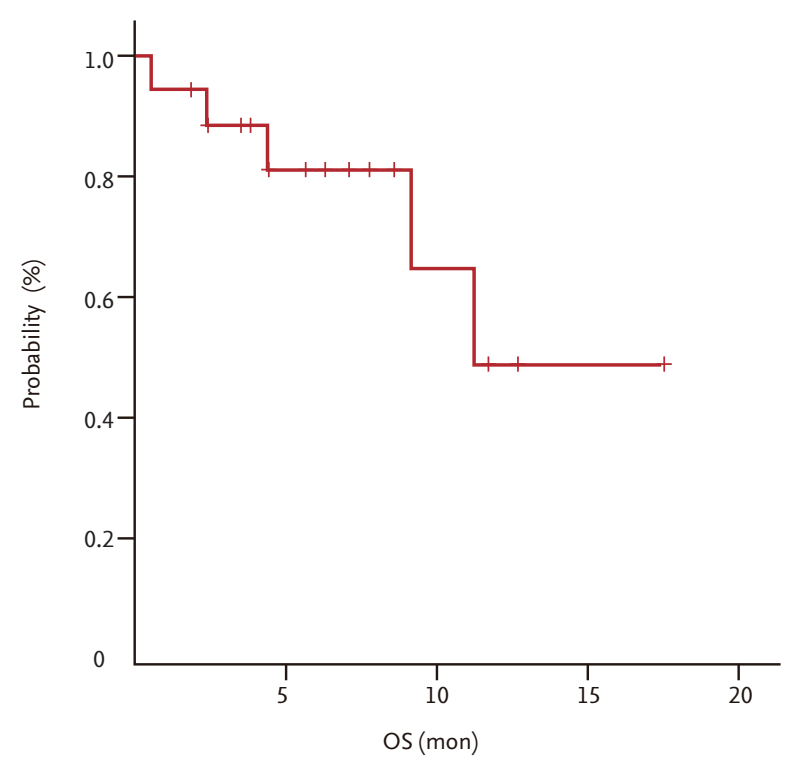

Figure 1. Kaplan-Meier survival curve for overall survival (OS).

in R/M HNSCC by showing a higher response rate (21\% to $32 \%)$ than that achieved with monotherapy (17\%), without survival gain $[19,20]$. Other chemotherapy doublets have also been evaluated as first-line treatment of R/M HNSCC [6-9,21-27]. Among them, a 3-week schedule of combined docetaxel and cisplatin was found to have remarkable efficacy despite its severe toxicities [69]. Glisson et al. [8] $(n=36)$ reported an ORR of $40 \%(6 \%$ CR, 34\% PR), a median PFS of 4 months, and an OS of 9.6 months for a triweekly docetaxel $75 \mathrm{mg} / \mathrm{m}^{2}$ and cisplatin $75 \mathrm{mg} / \mathrm{m}^{2}$ regimen. However, grade 4 neutropenia was observed in $71 \%$ of patients. Other grade 3 and 4 side effects included asthenia (25\%), nausea (11\%), fever (8\%), and diarrhea (8\%). Caponigro et al. [9] $(n=46)$ demonstrated an ORR of $46 \%$ (11\% CR, 35\% PR) and a median OS of 11 months for a 3-week docetaxel $75 \mathrm{mg} /$ $\mathrm{m}^{2}$ and cisplatin $100 \mathrm{mg} / \mathrm{m}^{2}$ regimen. However, a high incidence of grade 3-4 neutropenia (61\%) was also noted in this study. Given the high incidence of poor performance status, the high prevalence of comorbid conditions, and risk factors including smoking and alcoholism in patients with R/M HNSCC, chemotherapeutic agents with less toxicity should be developed. In this context, our regimen of cisplatin with weekly docetaxel is reasonable in terms of efficacy and toxicity.

It has been reported that East Asian ethnic groups experience more severe toxicities including myelosuppres-

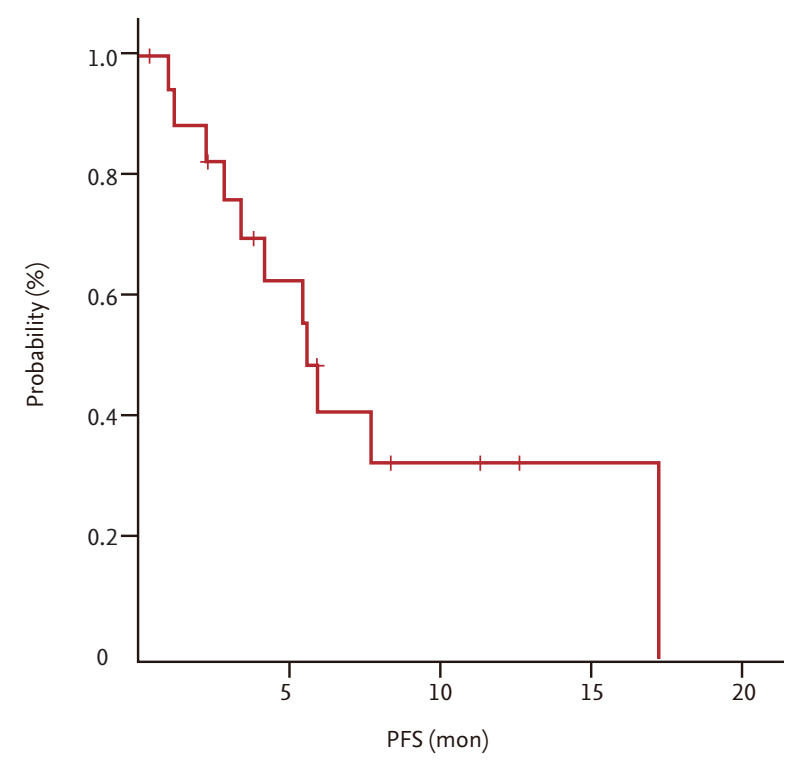

Figure 2. Kaplan-Meier survival curve for progression-free survival (PFS).

Table 3. Adverse events

\begin{tabular}{lll}
\hline Variable & Grade 1-2(\%) & Grade 3-4 (\%) \\
\hline Hematologic toxicities & & \\
Anemia & $9(50)$ & 0 \\
Leukopenia & $6(33.3)$ & $1(5.6)$ \\
Neutropenia & $8(44.4)$ & $1(5.6)$ \\
Thrombocytopenia & $2(11.1)$ & 0 \\
\hline Febrile neutropenia & $1(5.6)$ & 0 \\
\hline Non-hematologic toxicities & & \\
Diarrhea & $2(11.1)$ & 0 \\
\hline Oral mucositis & $4(22.2)$ & 0 \\
Infection (pneumonia, & $2(11.1)$ & $1(5.6)$ \\
sepsis) & & \\
\hline Interstitial lung disease & 0 & 0 \\
Asthenia & $2(11.1)$ & 0 \\
\hline Neuropathy & 0 & 0 \\
\hline Nail changes & 0 & 0 \\
\hline Hepatotoxicity & $4(22.2)$ & 0 \\
\hline
\end{tabular}

sion or mucositis with a 3-week schedule of docetaxel compared to Western ethnic groups [28-31]. This phenomenon is thought to be the result of pharmacogenomic differences between the two ethnic groups. According to previous studies, polymorphisms in cytochrome $\mathrm{P} 450$, family 3, subfamily A (CYP3A) isoenzymes, which inacti- 
vate docetaxel, are believed to play an important role in these pharmacokinetic differences. In East Asian patients, $\mathrm{CYP}_{3} \mathrm{~A}$ activity and docetaxel clearance are notably lower than those in Western patients [28-31]. Docetaxel causes characteristic early bone marrow suppression, which occurs 5 to 9 days after infusion, whereas this adverse effect appears 10 to 14 days after cisplatin infusion; thus, we hypothesized that splitting the docetaxel schedule would reduce overlapping toxicities compared to the 3 -week schedule of docetaxel and cisplatin [32].

Our group previously reported a very high response rate (70\%) and low hematologic or non-hematologic toxicities (no grade $3 / 4$ toxicity) with this combination regimen in patients with recurrent or metastatic nasopharyngeal cancer, even though the nature of the disease and patient characteristics are different [15]. Several studies regarding a regimen of weekly docetaxel as a single agent in the treatment of R/M HNSCC have demonstrated an excellent toxicity profile, particularly notable for a low rate of grade 3/4 neutropenia, ranging from $0 \%$ to $12.5 \%$, but a variable ORR (7\% to $42 \%$ ) and OS (17.9 weeks to 11.3 months) [33-36]. Three [33,35,36] of four studies showed suboptimal response rate and survival, but these differences could be explained by differences in the study populations, which included patients who received palliative chemotherapy as second-line or more for R/M HNSCC. Okamoto et al. [33] compared the efficacy and safety of weekly docetaxel at a dose of 25 to $30 \mathrm{mg} / \mathrm{m}^{2}$ with a triweekly docetaxel regimen of 60 $\mathrm{mg} / \mathrm{m}^{2}$. The ORR was $22 \%$ for the 18 patients (one pretreated) in the weekly docetaxel group, and $47.8 \%$ for the 29 patients (10 pretreated) in the triweekly docetaxel group. Only one (5.6\%) case of grade 3 mucositis developed in the group receiving weekly docetaxel, while 12 cases of grade 3 or 4 neutropenia (41.4) and two cases of grade 3 or 4 thrombocytopenia (6.9\%) occurred in the triweekly docetaxel group. Cho et al. [35] evaluated the efficacy and toxicity of weekly docetaxel at a dose of 35 $\mathrm{mg} / \mathrm{m}^{2}$ in patients with platinum-refractory R/M HNSCC and obtained an acceptable clinical outcome, with an ORR of $13 \%$ and an OS of 29 weeks ( $95 \%$ CI, 10.8 to 47.1) in the setting of second-line or further chemotherapy. Grade 3 neutropenia occurred in only $8.7 \%$ of patients. Specenier et al. [36] reported the lowest ORR of the studies cited, at $7 \%$, along with an OS of 17.9 weeks
(95\% CI, 10.1 to 25.6 ), in 30 patients receiving weekly docetaxel of $36 \mathrm{mg} / \mathrm{m}^{2}$, among whom 27 (90\%) were pretreated patients. There were no reported episodes of grade 4 neutropenia, thrombocytopenia, or non-hematological toxicity.

To the best of our knowledge, this is the first study to investigate the efficacy and acceptable toxicities of cisplatin doublets with weekly docetaxel as first-line treatment for patients with R/M HNSCC in Asia. Considering the small number of patients enrolled in this study, large randomized pharmacogenomic and epidemiologic studies would be helpful in establishing the basis of differences in toxicity and efficacy among patients with R/M HNSCC.

The present study has some limitations. First, this study was performed at a single institution with a small study population. Thus, a larger number of patients along with a comparative study are needed to confirm our results. Secondly, no information was available about tumor human papilloma virus (HPV) status, which may have contributed to heterogeneity in prognosis within the patient population. The prognostic impact of HPV on HNSCC has been evaluated in numerous studies over the past two decades, and HPV-positive status is widely known to be associated with a better prognosis [37-46]. In addition, the results of four phase III clinical trials have confirmed HPV tumor status as a predictor of prognosis regardless of treatment regimen [37-40].

In conclusion, the present study demonstrated that a regimen of cisplatin and weekly docetaxel was clinically efficacious and had tolerable toxicities as first-line chemotherapy in patients with R/M HNSCC.

\section{KEY MESSAGE}

1. The standard treatment for locally advanced head and neck squamous cell carcinoma varies worldwide.

2. For patients with recurrent, metastatic recurrent, or metastatic squamous cell carcinoma of the head and neck, a regimen of cisplatin and weekly docetaxel showed high efficacy with tolerable toxicity as a first-line treatment. 


\section{Conflict of interest}

No potential conflict of interest relevant to this article was reported.

\section{REFERENCES}

1. The World Cancer Report: the major findings. Cent Eur J Public Health 2003;11:177-179.

2. Kotwall C, Sako K, Razack MS, Rao U, Bakamjian V, Shedd DP. Metastatic patterns in squamous cell cancer of the head and neck. Am J Surg 1987;154:439-442.

3. Zbaren P, Lehmann W. Frequency and sites of distant metastases in head and neck squamous cell carcinoma. An analysis of 101 cases at autopsy. Arch Otolaryngol Head Neck Surg 1987;113:762-764.

4. Haddad RI, Shin DM. Recent advances in head and neck cancer. N Engl J Med 2008;359:1143-1154.

5. Jemal A, Siegel R, Ward E, et al. Cancer statistics, 2008. CA Cancer J Clin 2008;58:71-96.

6. Specht L, Larsen SK, Hansen HS. Phase II study of docetaxel and cisplatin in patients with recurrent or disseminated squamous-cell carcinoma of the head and neck. Ann Oncol 2000;11:845-849.

7. Schoffski P, Catimel G, Planting AS, et al. Docetaxel and cisplatin: an active regimen in patients with locally advanced, recurrent or metastatic squamous cell carcinoma of the head and neck. Results of a phase II study of the EORTC Early Clinical Studies Group. Ann Oncol 1999;10:119-122.

8. Glisson BS, Murphy BA, Frenette G, Khuri FR, Forastiere AA. Phase II trial of docetaxel and cisplatin combination chemotherapy in patients with squamous cell carcinoma of the head and neck. J Clin Oncol 2002;20:1593-1599.

9. Caponigro F, Massa E, Manzione L, et al. Docetaxel and cisplatin in locally advanced or metastatic squamous-cell carcinoma of the head and neck: a phase II study of the Southern Italy Cooperative Oncology Group (SICOG). Ann Oncol 2001;12:199-202.

10. Chen YM, Shih JF, Perng RP, Tsai CM, Whang-Peng J. A randomized trial of different docetaxel schedules in nonsmall cell lung cancer patients who failed previous platinum-based chemotherapy. Chest 2006;129:1031-1038.

11. Tabernero J, Climent MA, Lluch A, et al. A multicentre, randomised phase II study of weekly or 3-weekly docetaxel in patients with metastatic breast cancer. Ann
Oncol 2004;15:1358-1365.

12. Charles KA, Rivory LP, Stockler MR, et al. Predicting the toxicity of weekly docetaxel in advanced cancer. Clin Pharmacokinet 2006;45:611-622.

13. Hainsworth JD. Practical aspects of weekly docetaxel administration schedules. Oncologist 2004;9:538-545.

14. Ji JH; Korean Cancer Study Group (KCSG), Yun T, et al. A prospective multicentre phase II study of cisplatin and weekly docetaxel as first-line treatment for recurrent or metastatic nasopharyngeal cancer (KCSG HNo7-o1). Eur J Cancer 2012;48:3198-3204.

15. Kim YS, Sym SJ, Park SH, et al. A randomized phase II study of weekly docetaxel/cisplatin versus weekly docetaxel/oxaliplatin as first-line therapy for patients with advanced gastric cancer. Cancer Chemother Pharmacol 2014;73:163-169.

16. Therasse P, Arbuck SG, Eisenhauer EA, et al. New guidelines to evaluate the response to treatment in solid tumors. European Organization for Research and Treatment of Cancer, National Cancer Institute of the United States, National Cancer Institute of Canada. J Natl Cancer Inst 2000;92:205-216.

17. Pfister DG, Ang K, Brockstein B, et al. NCCN practice guidelines for head and neck cancers. Oncology (Williston Park) 2000;14:163-194.

18. Benson AB 3rd, Abrams TA, Ben-Josef E, et al. NCCN clinical practice guidelines in oncology: hepatobiliary cancers. J Natl Compr Canc Netw 2009;7:350-391.

19. Jacobs C, Lyman G, Velez-Garcia E, et al. A phase III randomized study comparing cisplatin and fluorouracil as single agents and in combination for advanced squamous cell carcinoma of the head and neck. J Clin Oncol 1992;10:257-263.

20. Forastiere AA, Metch B, Schuller DE, et al. Randomized comparison of cisplatin plus fluorouracil and carboplatin plus fluorouracil versus methotrexate in advanced squamous-cell carcinoma of the head and neck: a Southwest Oncology Group study. J Clin Oncol 1992;10:1245-1251.

21. Kucukzeybek Y, Gorumlu G, Karaca B, et al. Docetaxel and platinum combination chemotherapy in locally advanced or metastatic head and neck cancer. J BUON 2008;13:199203.

22. Argiris A, Buchanan A, Brockstein B, et al. Docetaxel and irinotecan in recurrent or metastatic head and neck cancer: a phase 2 trial of the Eastern Cooperative Oncology Group. Cancer 2009;115:4504-4513. 
23. Fayette J, Montella A, Chabaud S, et al. Paclitaxel is effective in relapsed head and neck squamous cell carcinoma: a retrospective study of 66 patients at a single institution. Anticancer Drugs 2010;21:553-558.

24. Luo Z, Chang J, Guo Y, et al. Continuous infusion of 5-FU with split-dose cisplatin: an effective treatment for advanced squamous-cell carcinoma of the head and neck. Clin Invest Med 2011;34:E8-E13.

25. Won YW, Park YH, Ahn MJ, Do IG, Ko YH, Park K. A phase II study of combination chemotherapy with capecitabine and cisplatin in patients with metastatic or recurrent squamous cell carcinoma of the head and neck. Ann Oncol 2011;22:417-423.

26. Huang YC, Chang PM, Chen MH, et al. A study using ifosfamide and etoposide in patients with cisplatin-refractory recurrent or metastatic head and neck squamous cell carcinoma. Jpn J Clin Oncol 2011;41:630-636.

27. Kafri Z, Heilbrun LK, Sukari A, et al. Phase II study of gemcitabine and docetaxel combination in patients with previously treated recurrent or metastatic squamous cell carcinoma of the head and neck. ISRN Oncol 2012;2012:159568.

28. Briasoulis E, Karavasilis V, Anastasopoulos D, et al. Weekly docetaxel in minimally pretreated cancer patients: a dose-escalation study focused on feasibility and cumulative toxicity of long-term administration. Ann Oncol 1999;10:701-706.

29. Miyata M, Yasuda K, Burioka N, et al. The influence of granisetron on the pharmacokinetics and pharmacodynamics of docetaxel in Asian lung cancer patients. Cancer J 2006;12:69-72.

30. Goh BC, Lee SC, Wang LZ, et al. Explaining interindividual variability of docetaxel pharmacokinetics and pharmacodynamics in Asians through phenotyping and genotyping strategies. J Clin Oncol 2002;20:3683-3690.

31. Bosch TM, Huitema AD, Doodeman VD, et al. Pharmacogenetic screening of $\mathrm{CYP}_{3} \mathrm{~A}$ and $\mathrm{ABCB} 1$ in relation to population pharmacokinetics of docetaxel. Clin Cancer Res 2006;12:5786-5793.

32. Cho SH, Go SI, Lee GW, et al. Phase II study of a biweekly schedule of docetaxel and cisplatin in patients with metastatic non-small cell lung cancer. Lung Cancer 2010;69:94-98.

33. Okamoto H, Yane K, Yamanaka T, Fukuda T, Hosoi H. Weekly docetaxel treatment for head and neck cancer. Gan To Kagaku Ryoho 2005;32:1915-1918.
34. Hitt R, Amador ML, Quintela-Fandino M, et al. Weekly docetaxel in patients with recurrent and/or metastatic squamous cell carcinoma of the head and neck. Cancer 2006;106:106-111.

35. Cho BC, Keum KC, Shin SJ, et al. Weekly docetaxel in patients with platinum-refractory metastatic or recurrent squamous cell carcinoma of the head and neck. Cancer Chemother Pharmacol 2009;65:27-32.

36. Specenier P, Rasschaert M, Vroman P, et al. Weekly docetaxel in patients with recurrent and/or metastatic squamous cell carcinoma of the head and neck. Am J Clin Oncol 2011;34:472-477.

37. Hong A, Dobbins T, Lee CS, et al. Relationships between epidermal growth factor receptor expression and human papillomavirus status as markers of prognosis in oropharyngeal cancer. Eur J Cancer 2010;46:2088-2096.

38. Ang KK, Harris J, Wheeler R, et al. Human papillomavirus and survival of patients with oropharyngeal cancer. $\mathrm{N}$ Engl J Med 2010;363:24-35.

39. Rischin D, Young RJ, Fisher R, et al. Prognostic significance of p16INK4A and human papillomavirus in patients with oropharyngeal cancer treated on TROG 02.02 phase III trial. J Clin Oncol 2010;28:4142-4148.

40. Hannisdal K, Schjolberg A, De Angelis PM, Boysen M, Clausen OP. Human papillomavirus (HPV)-positive tonsillar carcinomas are frequent and have a favourable prognosis in males in Norway. Acta Otolaryngol 2010;130:293-299.

41. Chaturvedi AK, Engels EA, Pfeiffer RM, et al. Human papillomavirus and rising oropharyngeal cancer incidence in the United States. J Clin Oncol 2011;29:4294-4301.

42. Lassen P, Eriksen JG, Krogdahl A, et al. The influence of HPV-associated p16-expression on accelerated fractionated radiotherapy in head and neck cancer: evaluation of the randomised DAHANCA 6\&7 trial. Radiother Oncol 2011;100:49-55.

43. Hong AM, Dobbins TA, Lee CS, et al. Use of cyclin D1 in conjunction with human papillomavirus status to predict outcome in oropharyngeal cancer. Int J Cancer 2011;128:1532-1545.

44. Sethi S, Ali-Fehmi R, Franceschi S, et al. Characteristics and survival of head and neck cancer by HPV status: a cancer registry-based study. Int J Cancer 2012;131:11791186.

45. Deng Z, Hasegawa M, Yamashita Y, et al. Prognostic value of human papillomavirus and squamous cell carcinoma 
antigen in head and neck squamous cell carcinoma. Cancer Sci 2012;103:2127-2134.

46. Andl T, Kahn T, Pfuhl A, et al. Etiological involvement of oncogenic human papillomavirus in tonsillar squamous cell carcinomas lacking retinoblastoma cell cycle control. Cancer Res 1998;58:5-13. 